\title{
Yiqi Huoxue preserves heart function by upregulating the Sigma-1 receptor in rats with myocardial infarction
}

\author{
LIXIA LOU ${ }^{1}$, CHUNHONG LI $^{1}$, JIE WANG ${ }^{2}$, AIMING WU ${ }^{1}$, TING ZHANG ${ }^{1}$, ZHE MA $^{1}$, LIMIN CHAI $^{1}$, \\ DONGMEI ZHANG ${ }^{1}$, YIZHOU ZHAO ${ }^{1}$, BO NIE ${ }^{1}$, QIUSHUO JIN ${ }^{1}$, HUIYANG CHEN ${ }^{1}$ and WEI JING LIU ${ }^{1}$ \\ ${ }^{1}$ Key Laboratory of Chinese Internal Medicine of Ministry of Education and Beijing, \\ Dongzhimen Hospital Affiliated to Beijing University of Chinese Medicine, Beijing 100700; \\ ${ }^{2}$ Department of Cardiology, Lanzhou New District First People's Hospital, Lanzhou, Gansu 730300, P.R. China
}

Received May 6, 2020; Accepted December 15, 2020

DOI: $10.3892 / \mathrm{etm} .2021 .10743$

\begin{abstract}
Yiqi Huoxue (YQHX) is widely used in traditional Chinese medical practice due to its reported cardioprotective effects. The aim of the present study was to investigate the mechanism underlying these effects of YQHX via the regulation of the Sigma-1 receptor. The Sigma-1 receptor is a chaperone protein located on the mitochondrion-associated endoplasmic reticulum (ER) membrane. It serves an important role in heart function by regulating intracellular $\mathrm{Ca}^{2+}$ homeostasis and enhancing cellular bioenergetics. In the present study, male Sprague Dawley rats with myocardial infarction (MI)-induced heart failure were used. MI rats were administered different treatments, including normal saline, $\mathrm{YQHX}$ and fluvoxamine, an agonist of the Sigma-1 receptor. Following four weeks of treatment, YQHX was revealed to improve heart function and attenuate myocardial hypertrophy in MI rats. Additionally, YQHX increased the ATP content and improved the mitochondrial ultrastructure in the heart tissues of MI rats in comparison with acontrol. Treatment was revealed to attenuate the decreased expression of the Sigma-1 receptor and increase the expression of inositol triphosphate type 2 receptors $\left(\mathrm{IP}_{3} \mathrm{R} 2\right)$ in $\mathrm{MI}$ rats. By exposing $\mathrm{H} 9 \mathrm{c} 2$ cells to angiotensin II (Ang II), YQHX prevented cell hypertrophy and normalized the decreased ATP content. However, these positive effects were partially inhibited when the Sigma-1 receptor was knocked down via small interfering RNA transfection. The results of the present study suggested that the Sigma-1 receptor serves an important role in the cardioprotective efficacy of YQHX by increasing ATP content and attenuating cardiomyocyte hypertrophy.
\end{abstract}

Correspondence to: Dr Wei Jing Liu, Key Laboratory of Chinese Internal Medicine of Ministry of Education and Beijing, Dongzhimen Hospital Affiliated to Beijing University of Chinese Medicine, 5 Haiyuncang, Dongcheng, Beijing 100700, P.R. China E-mail: liuweijing-1977@hotmail.com

Key words: heart failure, Yiqi Huoxue, Sigma-1 receptor, cardiomyocyte hypertrophy, ATP

\section{Introduction}

Heart failure (HF) is the end-stage of numerous heart diseases, presenting a substantial clinical and economic burden on public health on a global scale. Due to an increase in the proportion of the aging population, the prevalence of HF is likely to increase in the future. Though numerous efforts have been made in cardiovascular research, current therapeutic approaches do not improve the mortality and readmission rates of patients with HF (1). In Chinese clinical practice, formulations from traditional Chinese medicine that have been demonstrated to be effective in improving heart function are used extensively. However, the mechanisms underlying these improvements has yet to be fully elucidated.

Various mechanisms have been demonstrated to be involved in the pathophysiological development of HF, including neuro-endocrine, inflammatory and structural remodeling mechanisms (2-4). The energy starvation hypothesis for HF has attracted particular attention (5). Recently, the Sigma-1 receptor (Sigma-1R), a molecular chaperone located on the endoplasmic reticulum (ER) membrane, was suggested to have a cardioprotective effect, potentially through energy metabolism improvement $(6,7)$. Sigma receptors were first reported in the central nervous system (CNS) as a subclass of opioid receptors. However, pharmacological and behavioral studies have revealed that sigma receptors are distinct from all previously characterized mammalian proteins. Sigma receptors are now recognized as non-opioid receptors located at the ER-mitochondrion interface, known as the mitochondrion-associated ER membrane (MAM) (8). While Sigma-1Rs have been extensively studied in the CNS, they are also found in multiple tissues, including the cardiovascular system. In fact, the protein levels of Sigma-1R have been revealed to be more highly expressed in the heart tissue than the brain tissue in rats (9). In the first study on Sigma-1R function in the heart, Sigma-1R stimulation was found to increase the contractility of cultured cardiomyocytes (10). Sigma-1R is responsible for mitochondrial metabolic regulation by regulating $\mathrm{Ca}^{2+}$ signaling from the ER into the mitochondrion (11). Tagashira et al (12) demonstrated that the upregulation of Sigma-1R by agonists in vivo may rescue cardiac dysfunction in mice with transverse aortic constriction-induced myocardial dysfunction, as well 
as in abdominal aortic constriction-induced cardiac hypertrophy ovariectomized female rats (13). The mechanisms of cardioprotection by Sigma-1Rs are the Sigma-1R-mediated Akt-eNOS pathway stimulation and $\mathrm{IP}_{3} \mathrm{R}$-mediated mitochondrial ATP production recovery $(12,13)$.

In traditional Chinese medicine the formulation Yiqi Huoxue (YQHX), is used to improve symptoms in patients with HF $(14,15)$. YQHX is thought to act through the regulation of mitochondrial function and energy metabolism (16). Qiliqiangxin is a widely used YQHX formula that has been reported to increase the ATP content in the heart tissue of HF rats (17). Similarly, Sigma-1R has been revealed to exert cardioprotective functions, including enhanced mitochondrial ATP production. However, whether Sigma-1R is also regulated by $\mathrm{YQHX}$ during HF treatment, in addition to its involvement in the enhancement of ATP in HF patients, requires further study. In the present study, a YQHX formulation comprising the following six types of Chinese herbs was used: Astragalus, Codonopsis pilosula, Salvia miltiorrhiza, Ligusticum wallichii, red Paeonia and carthami flowers (18). To investigate the role of Sigma-1R in the treatment of HF using YQHX, rats with myocardial infarction (MI)-induced HF and cultured $\mathrm{H} 9 \mathrm{c} 2$ cells with angiotensin II (Ang II)-induced hypertrophy were used to investigate the effect of YQHX on MI-induced myocardial dysfunction and Ang II-induced hypertrophy.

\section{Materials and methods}

Materials. YQHX was composed of $40 \mathrm{~g}$ astragalus, $40 \mathrm{~g}$ Codonopsis pilosula, $20 \mathrm{~g}$ Salvia miltiorrhiza, $20 \mathrm{~g}$ Ligusticum wallichii, $20 \mathrm{~g}$ red Paeonia and $20 \mathrm{~g}$ carthami flowers. Slices of the herbs were purchased from Beijing Tong Ren Tang Chinese Medicine Co., Ltd. The herbs were boiled in water for $1 \mathrm{~h}$ and aliquots at a final concentration of $3 \mathrm{~g}$ herbs/ml were stored at $4^{\circ} \mathrm{C}$. Fluvoxamine (Flv), an agonist of Sigma-1R, was purchased from Abbott Healthcare Products Ltd. Ang II was purchased from Chinese Peptide Company Ltd. Sigma-1R siRNA was provided by Sangon Biotech Co., Ltd.

DeterminationofYQHXcomponentsbyultra-high-performance liquid chromatography-mass spectrometry (UHPLC-MS). Aliquots of $0.02 \mathrm{~g}$ of lyophilized, pulverized, and homogenized herbs were extracted using $5 \mathrm{ml}$ methanol-water $(\mathrm{v}-\mathrm{v}=1: 1)$ for $20 \mathrm{~min}$ at room temperature. The extract was filtered through a $0.22-\mu \mathrm{m}$ membrane. Next, $3 \mu \mathrm{l}$ extract was injected into an analytical column (Shimadzu UHPLC system; Shimadzu Corporation), equipped with an LC-30AD solvent delivery system, an SIL-30AC autosampler, a CTO-30A column oven, a DGU-20A3 degasser, and a CBM-20A controller, for analysis. The separation of the compounds was performed using a Phenomenex Kinetex C18 $(2.6 \mu \mathrm{m}, 100 \times 2.1 \mathrm{~mm}$; Phenomenex Inc.) at $35^{\circ} \mathrm{C}$. The mobile phase, which consisted of $0.1 \%$ formic acid in water (A) and methanol (B), was delivered at a flow rate of $0.4 \mathrm{ml} / \mathrm{min}$ under a gradient program. The gradient system was as follows: $0-1 \mathrm{~min}, 5 \% \mathrm{~B}$; $1-35 \mathrm{~min}, 5-60 \% \mathrm{~B} ; 35-40 \mathrm{~min}$, $60-90 \% \mathrm{~B} ; 40-45 \mathrm{~min}, 90 \% \mathrm{~B} ; 45.0-45.1 \mathrm{~min}, 90-5 \% \mathrm{~B}$; 45.1-50 min, $5 \%$ B. The diode-array detector was set at $254 \mathrm{~nm}$ and the online UV spectra were recorded in a scanning range of $190-400 \mathrm{~nm}$.
The mass spectra were acquired using a TripleTOF ${ }^{\mathrm{TM}} 4600$ system with a Duo Spray source (SCIEX; AB Sciex Pte. Ltd.) in negative and positive ESI mode. The optimized parameters for the negative and positive modes were as follows: Ion spray voltage, 5,500 (positive ion mode) and -4,500 $\mathrm{V}$ (negative ion mode); Turbo V spray temperature, $600^{\circ} \mathrm{C}$; nebulizer gas (Gas 1), 50 psi; heater gas (Gas 2), 60 psi; collision gas, medium; curtain gas, 30 psi; declustering potential, 80 (positive ion mode) and $-80 \mathrm{~V}$ (negative ion mode). The collision energy was set at 35 (positive ion mode) and $-35 \mathrm{~V}$ (negative ion mode) with a spread of $15 \mathrm{~V}$ for the MS-MS experiments. The data were acquired with the information-dependent acquisition method. For TOF-MS and TOF-MS-MS analysis, the spectra covered the range from $\mathrm{m}-\mathrm{z} 70$ to 1,000 $\mathrm{Da}$ and 50-1,000 Da. The data were analyzed in Peak View Software ${ }^{\mathrm{TM}} 2.2$ (SCIEX).

Animal models with MI and treatments. The present study was approved by the Experimental Animal Ethics Committee of Dongzhimen Hospital, Beijing University of Chinese Medicine (ethical approval no. 16-24). Male adult Sprague-Dawley (SD) rats (age, 6-7 weeks; weight, 190-210 g) were obtained from Beijing Vital River Laboratory Animal Technology Co., Ltd. All the rats were kept in the barrier environment animal room of the Chinese Medicine Pharmacology Laboratory, Dongzhimen hospital [license no. SYXK(Beijing)2015-0001]. The animals were fed a standard laboratory diet and given free access to tap water. The cages were kept in a room with controlled temperature of $22 \pm 1^{\circ} \mathrm{C}$, relative humidity of $65-70 \%$ and on a $12 \mathrm{~h}$ light-dark cycle. A total of 40 rats were used in the study. All animals underwent a myocardial infarction operation or sham operation, as previously described (18). The rats in the control group underwent a sham operation with no left anterior descending coronary artery ligation. Following the surgical operation, 4 rats died and the surviving MI rats were divided randomly into three groups ( 9 rats in each group): MI ( $5 \mathrm{ml} / \mathrm{kg} / \mathrm{d}$ normal saline by gavage); MI + YQHX (5 ml/kg/d by gavage) and MI + Flv (1 mg/kg/d by gavage). Rats that had undergone a sham operation were administered with $5 \mathrm{ml} / \mathrm{kg} / \mathrm{d}$ normal saline by gavage. Treatments were administered on the day following surgery once a day for 4 weeks.

Echocardiography and measurement of cardiac hypertrophy. Non-invasive echocardiographic measurements were performed in the rats using a Sino-Japanese Joint AloCa5000 ultrasound system equipped with a 7-15 $\mathrm{MHz}$ real-time microvisualization scan head probe. In brief, after 4 weeks of treatment, each rat was anesthetized with sodium pentobarbital (50 mg/kg body weight), and their hearts were imaged in two-dimensional parasternal long-axis view. The left ventricular end-diastolic volume (LVEDV), left ventricular end-systolic volume (LVESV), left ventricular ejection fraction (LVEF), and left ventricular fractional shortening (LVFS) were measured. To determine the extent of cardiac hypertrophy, the hearts of each rat were weighed and the ratio of heart weight-to-body weight (HW/BW) was calculated.

Transmission electron microscopy (TEM). Following heart perfusion with $4 \%$ paraformaldehyde, the heart tissues were minced into 1-2 $\mathrm{mm}^{3}$ cubes and fixed with $2 \%$ glutaraldehyde in $0.1 \mathrm{M}$ cacodylate buffer for $2 \mathrm{~h}$ at room temperature. Tissues 
were post-fixed with $2 \%$ osmium tetroxide for $1 \mathrm{~h}$ at $4^{\circ} \mathrm{C}$, and then rinsed three times with cold PBS. The tissues were then progressively dehydrated using a series of ethanol washes $(50,70,90$ and $100 \% \mathrm{EtOH})$, followed by dehydration with propylene oxide and infiltration with 1:1 propylene oxide:Epon with $1.5 \%$ DMP-30 for $1 \mathrm{~h}$. Finally, the tissues were cut into 70-nm sections using an ultramicrotome, placed on TEM grids, stained with lead citrate, and imaged using a transmission electron microscope (Hitachi Ltd.; model H-7650).

Measurement of ATP content in heart tissue. Heart tissues were collected 4 weeks post-surgery and homogenized on ice prior to assaying for ATP content. The ATP content was determined using an ATP colorimetric-fluorometric assay kit (cat. no. MAK1900; Sigma-Aldrich; Merck KGaA), according to the manufacturer's protocol. In brief, heart tissue $(10 \mathrm{mg})$ was homogenized in $100 \mu \mathrm{l}$ ATP assay buffer and then deproteinized using a 10-kDa MWCO spin filter. ATP standards and fluorometric reaction mixtures were prepared according to the manufacturer's protocol. Next, fluorometric reaction mixtures with standards or samples were incubated in the plate at room temperature for $30 \mathrm{~min}$ in the dark. The fluorescence (FLU; $\lambda_{\text {ex }}=535 \mathrm{~nm}$ and $\lambda_{\mathrm{em}}=587 \mathrm{~nm}$ ) was measured using a microplate reader and the amount of ATP in the samples was calculated using an ATP standard curve.

Western blot analysis. Western blot analysis was performed as previously described (18). The heart tissues were homogenized on ice in RIPA buffer with complete protease inhibitor (Roche Diagnostics $\mathrm{GmbH}$ ). Following centrifugation for $20 \mathrm{~min}$, the protein concentration of the resulting clear supernatant was determined using the BCA method before use for western blotting. In brief, 40-100 $\mu \mathrm{g}$ proteins were loaded and separated by 10 or $8 \%$ SDS-PAGE and transferred onto nitrocellulose membranes. Membranes were blocked in Tris-buffered saline-Tween-20 (TBST) with 5\% skimmed milk at room temperature for $1 \mathrm{~h}$, and then incubated with primary antibodies overnight at $4^{\circ} \mathrm{C}$. Following rinsing, the membranes were incubated with peroxidase conjugated secondary antirabbit (dilution, 1:5,000; cat. no. BA1054; Boster Biological Technology Co., Ltd.) or antimouse antibodies (dilution, 1:5,000; cat. no. BA1050; Boster Biological Technology Co., Ltd.) at room temperature for $1 \mathrm{~h}$ and evaluated by enhanced chemiluminescence (Super ECL Plus; Applygen Technologies) using a chemiluminescence image analysis system (Tanon 4600; Tanon Science and Technology Co., Ltd). Quantification was performed with densitometric analysis using NIH ImageJ software (version 1.29; National Institutes of Health). The antibodies used included anti-Sigma-1 receptor antibody (dilution, 1:1,000; cat. no. 61994; Cell Signaling Technology, Inc.), anti-inositol-1,4,5-trisphosphate type 2 receptor $\left(\mathrm{IP}_{3} \mathrm{R} 2\right)$ antibody (dilution,1:500; cat. no. sc-398434; Santa Cruz Biotechnology, Inc.) and anti-GAPDH antibody (1:5,000; cat. no. ab181602; Abcam).

Reverse transcription-quantitative polymerase chain reaction ( $R T-q P C R)$. Total RNA was extracted using TRIzol ${ }^{\circledR}$ reagent, according to the manufacturer's protocol (Invitrogen; Thermo Fisher Scientific, Inc.). Next, reverse transcription was performed using $1 \mu \mathrm{g}$ total RNA and RevertAid Reverse
Transcriptase and oligo(dT) $)_{18}$ (cat. no. K1622; Thermo Fisher Scientific, Inc.) at the following thermal profile: $42^{\circ} \mathrm{C}$ for $60 \mathrm{~min}$ and $70^{\circ} \mathrm{C}$ for $5 \mathrm{~min}$. PCR reactions were performed using SYBR green master mix (Applied Biosystems; Thermo Fisher Scientific, Inc.) in a PCR machine (MxPro-Mx3000P; Agilent Technologies, Inc.) using the following specific primer pairs: Calcineurin A (forward, 5'-CGATTCTCCGACAGG AAAAA-3' and reverse, 5'-AAGGCCCACAAATACAGC AC-3'); brain natriuretic peptide (BNP; forward, 5'-CAGAAG CTGCTGGAGCTGATA-3' and reverse, 5'-TCCGGTCTATCT TCTGCCCA-3'); and GAPDH (forward, 5'-AGTTCAACG GCACAGTCAAG-3' and reverse, 5'-TACTCAGCACCAGCA TCACC-3'). The thermocycling conditions were as follows: Denaturation at $95^{\circ} \mathrm{C}$ for $10 \mathrm{~min}$, followed by 40 cycles of $95^{\circ} \mathrm{C}$ for $30 \mathrm{sec} 55^{\circ} \mathrm{C}$ for $30 \mathrm{sec}$ and $72^{\circ} \mathrm{C}$ for $20 \mathrm{sec}$. Data analysis was performed using MxPro-Mx3000P software, and was calculated as the change in the cycle threshold $(\triangle \mathrm{CT})$ for the target gene relative to the $\triangle \mathrm{CT}$ for GAPDH (control gene) (19).

$H 9 c 2$ cell culture and small interfering RNA (siRNA) transfection. The H9c2 cell line was obtained from China National Infrastructure of Cell Line Resource and maintained in plastic $25-\mathrm{cm}^{2}$ flasks in Dulbecco's modified Eagle's medium (DMEM-F12; Biological Industries), containing 10\% fetal bovine serum (FBS). The culture flasks were kept in a $95 \%$ air and $5 \% \mathrm{CO}_{2}$ humidified environment at $37^{\circ} \mathrm{C}$. At confluence, the cells were washed and maintained in serum-free DMEM without supplements for $24 \mathrm{~h}$ prior to the subsequent experiments. The cells were then cultured with Ang II $\left(10^{-7} \mathrm{~mol} / \mathrm{l}\right)$ or YQHX (freeze-dried powder of YQHX dissolved in DMEM, $0.1 \mathrm{mg} / \mathrm{ml}$ ) for $72 \mathrm{~h}$ prior to being harvested. To silence Sigma-1R expression, H9c2 cells were transfected with Sigma-1R siRNA (sense, 5'-ACACGTGGATGGTGG AGTA-3' and anti-sense, 5'-TACTCCACCATCCACGTGT-3'). The negative control siRNA (sense, 5'-TTCTCCGAACGT GTCACGT-3' and anti-sense, 5'-ACGTGACACGTTCGG AGAA-3'; Sangon Biotech Co., Ltd.) was used as control. In brief, according to manufacturer's protocols, the cultured cells were plated onto 6 -well culture plates with $2.5 \mathrm{ml}$ medium per well. Next, $4.6 \mu \mathrm{l}$ Sigma-1R siRNA $(20 \mu \mathrm{M})$ was added to $125 \mu \mathrm{l}$ DMEM and $7.5 \mu \mathrm{l}$ Lipofectamine 2000 (GeneCopoeia Inc.) was added to $125 \mu \mathrm{l}$ DMEM. The two solutions were incubated separately at room temperature for $5 \mathrm{~min}$ prior to mixing and incubating at room temperature for $15 \mathrm{~min}$. The resulting solution was added to cells, which were incubated at $37^{\circ} \mathrm{C}$ in a $5 \% \mathrm{CO}_{2}$ atmosphere for $6 \mathrm{~h}$ to initiate transfection. The medium was replaced with complete medium and the transfected cells were collected for detection at $24 \mathrm{~h}$ or $72 \mathrm{~h}$ after transfection. In the control group, the $\mathrm{H} 9 \mathrm{c} 2$ cells were transfected with a negative control siRNA for $6 \mathrm{~h}$ at $37^{\circ} \mathrm{C}$ in a $5 \% \mathrm{CO}_{2}$ atmosphere using Lipofectamine 2000.

Cell surface area measurement. To establish the cell surface area, H9c2 cells were stained with rhodamine-conjugated phalloidin (Beijing Solarbio Science \& Technology Co., Ltd.). According to the manufacturer's protocols, the cultured cells were washed twice with $37^{\circ} \mathrm{C}$ PBS and then fixed in $4 \%$ formaldehyde for $10 \mathrm{~min}$ at room temperature. Following permeabilizing in $0.1 \%$ Triton X-100 in PBS for $5 \mathrm{~min}$ at room temperature, the cells were incubated with 


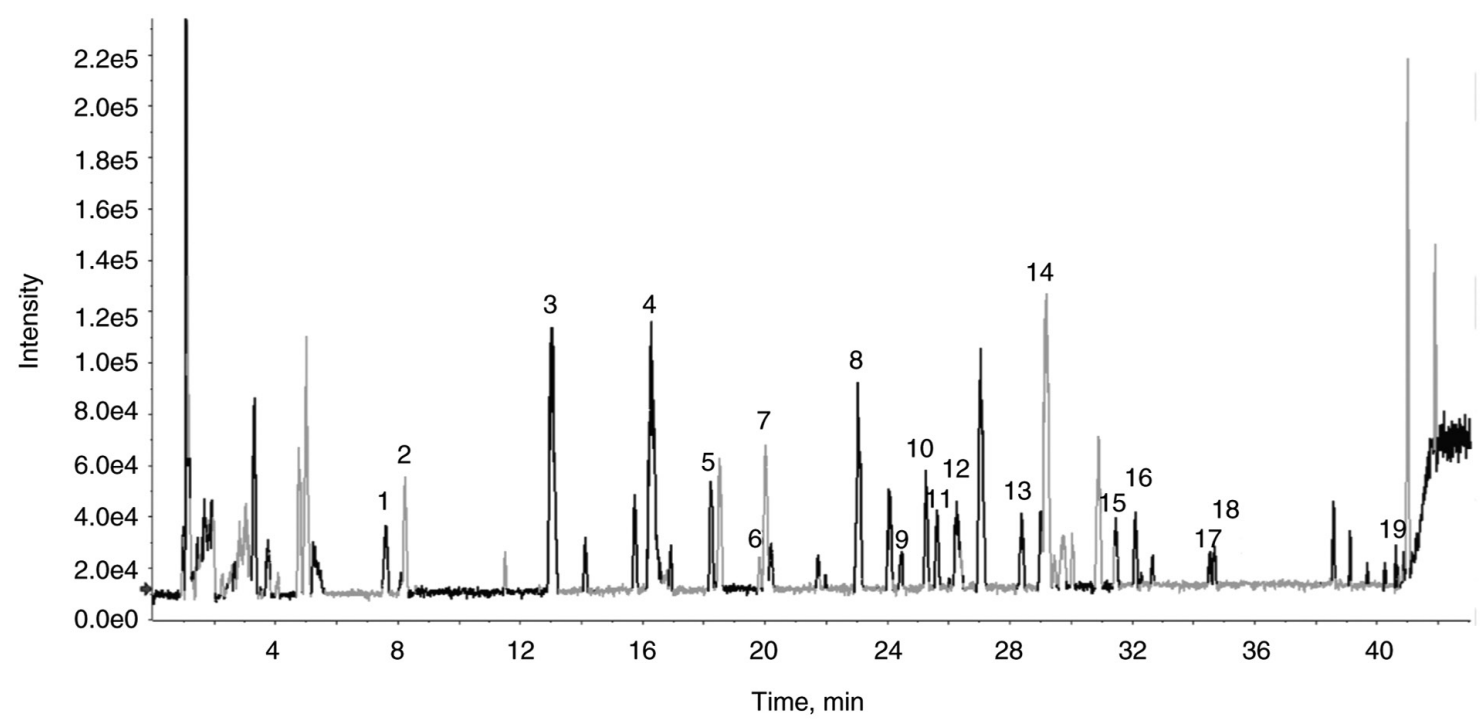

Figure 1. Base peak chromatogram of electrospray ionization (+) time of flight-mass spectrometry from YQHX extracts. YQHX, Yiqi Huoxue.

rhodamine-conjugated phalloidin $(100 \mathrm{nM})$ at room temperature for $30 \mathrm{~min}$ in the dark. Next, the nuclei were stained with DAPI for $30 \mathrm{sec}$ and images of H9c2 cells were captured using a laser confocal microscope at 400x magnification (TCS SP8X; Leica Microsystems, Inc.). The surface areas were then analyzed using Image-Pro Plus 6.0 software (Media Cybernetics, Inc.). Each group of cells was counted in six wells, and at least 100 cells were measured in each well. The cell surface area rate was given as the rate in the control group.

Measurement of ATP content in H9c2 cells. The ATP content in the $\mathrm{H} 9 \mathrm{c} 2$ cells was measured using an enhanced ATP detection kit (Beyotime Institute of Biotechnology). According to the manufacturer's protocols, the cultured cells were washed twice with PBS and lysed. After 5 min of centrifugation at $12,000 \mathrm{x} \mathrm{g}$ at $4^{\circ} \mathrm{C}$, the resulting supernatant was used for detection. Next, $100 \mu 1$ ATP detection fluid was added to the detection platform. The plate was left to stand at room temperature for 5 min to allow all the background ATP to be consumed. Next, $20 \mu \mathrm{l}$ the sample or standard was added to each detection platform and mixed quickly. Finally, the mixture was analyzed using a luminometer (Varioscan Flash; Thermo Fisher Scientific, Inc.).

Statistical analysis. All of the statistical tests were performed using SPSS 16.0 (SPSS, Inc.). All values are expressed as the mean \pm standard deviation. The statistical significance of the differences between the groups was tested using one-way analysis of variance, followed by the Tukey's post hoc test. Two group comparisons were performed using independent sample t-tests. Comparisons of non-normal distribution data were followed by analysis with the Kruskal-Wallis method. $\mathrm{P}<0.05$ was considered to indicate a statistically significant difference.

\section{Results}

Analysis of YQHX using liquid chromatography-mass spectrometry $(L C-M S)$. The main components of $\mathrm{YQHX}$ were analyzed by LC-MS. The typical base peak chromatograms of the components of YQHX are shown in Fig. 1. Approximately 21 major compounds were detected (Table I), including paeoniflorin, calycosin and tanshinone.

$Y Q H X$ preserves heart function and alleviates myocardial hypertrophy in MI rats. To observe the effect of YQHX on the heart function, non-invasive echocardiographic measurements were taken 4 weeks post-treatment. The results demonstrated that 4 weeks after MI surgery, the rats developed heart dysfunction, as shown by increased LVEDV and LVESV and decreased LVEF and LVFS (all $\mathrm{P}<0.01$ vs. sham; Table II). YQHX administration for 4 weeks attenuated the impairment of LVEF and LVFS (all P<0.01 vs. MI; Table II) but had no effects on LVEDV and LVESV (Table II). Hypertrophy markers, including the ratio of $\mathrm{HW} / \mathrm{BW}$, and the levels of calcineurin A and BNP mRNA were also detected. The ratio of HW/BW was found to increase in the MI group, compared with the sham-operated controls ( $\mathrm{P}<0.01$ vs. sham; Fig. $2 \mathrm{~A}$ ). YQHX treatment significantly decreased the rise in the HW/BW ratio ( $\mathrm{P}<0.01$ vs. sham; Fig. $2 \mathrm{~A})$, and a similar effect was observed in Flv (Fig. 2A). The calcineurin A and BNP mRNA levels were significantly increased in the MI group, and YQHX and Flv treatment antagonized the increase in calcineurin $\mathrm{A}$ and BNP mRNA levels (Fig. 2C and D).

YQHX improves the ATP content and mitochondrial ultrastructure of myocardial tissue in MI rats. To observe the effect of YQHX on energy metabolism in HF, the ATP content was detected in the hearts of the different groups. The results demonstrated that 4 weeks after MI surgery, the ATP content in the rats was found to decrease to $53 \%$ in the heart tissues, compared with the sham controls $(\mathrm{P}<0.05$; Fig. 3$)$. YQHX administration increased the ATP content in the heart tissues to nearly normal levels $(\mathrm{P}<0.05$; Fig. 3). Flv treatment exhibited a tendency to increase the ATP content in heart failure ( $\mathrm{P}>0.05$; Fig. 3).

Approximately $90 \%$ of ATP is synthesized inside the mitochondria. The function of mitochondria is based on its structure. Therefore, the mitochondrial ultrastructure of the 
Table I. Components identified from YQHX extracts.

\begin{tabular}{|c|c|c|c|}
\hline Peak no. & Retention time, $\min$ & Assigned identity & Molecular formula \\
\hline 1 & 7.6 & Dihydrokaempferol-3-O-glucoside & $\mathrm{C}_{21} \mathrm{H}_{22} \mathrm{O}_{11}$ \\
\hline 2 & 8.2 & Tryptophan & $\mathrm{C}_{11} \mathrm{H}_{12} \mathrm{~N}_{2} \mathrm{O}_{2}$ \\
\hline 3 & 13 & Hydroxysafflor yellow A & $\mathrm{C}_{27} \mathrm{H}_{32} \mathrm{O}_{16}$ \\
\hline 4 & 16.3 & Paeoniflorin & $\mathrm{C}_{23} \mathrm{H}_{28} \mathrm{O}_{11}$ \\
\hline 5 & 18.44 & Ferulic acid & $\mathrm{C}_{10} \mathrm{H}_{10} \mathrm{O}_{4}$ \\
\hline 6 & 19.6 & Senkyunolide J & $\mathrm{C}_{12} \mathrm{H}_{18} \mathrm{O}_{4}$ \\
\hline 7 & 20.1 & Calycosin-7-O- $\beta$-D-glucoside & $\mathrm{C}_{22} \mathrm{H}_{22} \mathrm{O}_{10}$ \\
\hline 8 & 23.1 & 4-hydroxy-3-butylphthalide & $\mathrm{C}_{12} \mathrm{H}_{14} \mathrm{O}_{3}$ \\
\hline 9 & 24.4 & Senkyunolide F & $\mathrm{C}_{12} \mathrm{H}_{14} \mathrm{O}_{3}$ \\
\hline 10 & 25.6 & Ononin & $\mathrm{C}_{22} \mathrm{H}_{22} \mathrm{O}_{9}$ \\
\hline 11 & 25.7 & Lobetyolin & $\mathrm{C}_{20} \mathrm{H}_{28} \mathrm{O}_{8}$ \\
\hline 12 & 26.2 & Salvianlic acid B & $\mathrm{C}_{36} \mathrm{H}_{30} \mathrm{O}_{16}$ \\
\hline 13 & 28.3 & 3-butylidenephthalide & $\mathrm{C}_{12} \mathrm{H}_{12} \mathrm{O}_{2}$ \\
\hline 14 & 29.1 & Calycosin & $\mathrm{C}_{16} \mathrm{H}_{12} \mathrm{O}_{5}$ \\
\hline 15 & 31.5 & benzoylpaeoniflorin & $\mathrm{C}_{30} \mathrm{H}_{32} \mathrm{O}_{12}$ \\
\hline 16 & 32.1 & Z-Ligustilide & $\mathrm{C}_{12} \mathrm{H}_{14} \mathrm{O}_{2}$ \\
\hline 17 & 34.5 & Senkyunolide A & $\mathrm{C}_{12} \mathrm{H}_{16} \mathrm{O}_{2}$ \\
\hline 18 & 34.6 & Formononetin & $\mathrm{C}_{16} \mathrm{H}_{12} \mathrm{O}_{4}$ \\
\hline 19 & 40.6 & Levistolide A & $\mathrm{C}_{24} \mathrm{H}_{28} \mathrm{O}_{4}$ \\
\hline 20 & 40.9 & Tanshinone I & $\mathrm{C}_{18} \mathrm{H}_{12} \mathrm{O}_{3}$ \\
\hline 21 & 41.9 & Tanshinone IIA & $\mathrm{C}_{19} \mathrm{H}_{18} \mathrm{O}_{3}$ \\
\hline
\end{tabular}

Table II. Echocardiographic data for rats 4 weeks after MI with treatments, including YQHX.

\begin{tabular}{lcccc}
\hline Parameter & Sham & MI & MI+YQHX & MI+Flv \\
\hline LVEDV & $220.1 \pm 43.3$ & $415.4 \pm 67.5^{\mathrm{a}}$ & $445.1 \pm 61.1^{\mathrm{a}}$ & $403.5 \pm 114.5^{\mathrm{a}}$ \\
LVESV & $24.8 \pm 8.9$ & $286.7 \pm 54.5^{\mathrm{a}}$ & $242.3 \pm 54.6^{\mathrm{a}}$ & $227.8 \pm 90.1^{\mathrm{a}}$ \\
LVEF & $90.4 \pm 3.3$ & $31.2 \pm 4.6^{\mathrm{a}}$ & $46.1 \pm 6.5^{\mathrm{a}, \mathrm{b}}$ & $45.7 \pm 11^{\mathrm{a}, \mathrm{b}}$ \\
LVFS & $63.2 \pm 5.2$ & $15.5 \pm 2.5^{\mathrm{a}}$ & $24.2 \pm 4.1^{\mathrm{a}, \mathrm{b}}$ & $24.1 \pm 7.2^{\mathrm{a}, \mathrm{b}}$ \\
\hline
\end{tabular}

${ }^{\mathrm{a}} \mathrm{P}<0.01$ vs. sham group. ${ }^{\mathrm{b}} \mathrm{P}<0.01$ vs. MI group. Values are presented as the mean \pm standard deviation $(\mathrm{n}=8)$. MI, myocardial infarction; YQHX, Yiqi Huoxue. recipe; Flv, fluvoxamine.LVEDV, left ventricular end-diastolic volume; LVESV, left ventricular end-systolic volume; LVEF, left ventricular ejection fraction; LVFS, left ventricular fractional shortening.

surrounding infarction areas of left ventricular (LV) myocardial tissues from the different groups was investigated using TEM. Representative micrographs are provided in Fig. 4. The mitochondria in the heart tissues of the sham group showed complete membranes and neatly arranged internal ridges. By contrast, in the MI rats, most of the mitochondrial membranes disappeared and the internal ridges were ruptured and indistinct. Subsequently, YQHX and Flv treatment were revealed to improve the mitochondrial ultrastructure of heart tissues following MI surgery (Fig. 4).

$Y Q H X$ administration attenuates the change in Sigma-1R and $I P_{3} R 2$ expression in the LV of MI rats. To evaluate the role of Sigma-1R in the curative effect of YQHX on HF, Sigma-1R and $\mathrm{Ca}^{2+}$ transporter-IP ${ }_{3} \mathrm{R} 2$ expression was detected in the heart tissues of rats by western blotting. The data showed that
Sigma-1R expression in the heart tissue decreased significantly 4 weeks post-MI surgery in rats $(\mathrm{P}<0.05$ vs. sham) and was be reversed by treatment with the YQHX and Sigma-1R agonist Flv ( $\mathrm{P}<0.01$ vs. MI; Fig. 5A). $\mathrm{IP}_{3} \mathrm{R} 2$ was upregulated 4 weeks post-MI surgery. However, the administration of YQHX and Flv eliminated this MI-induced upregulation of $\mathrm{IP}_{3} \mathrm{R} 2$ (Fig. 5B).

YQHX attenuates cell hypertrophy and decreases ATP induced by Ang II in H9c2 by stimulating Sigma-1R. Ventricular hypertrophy is a compensatory attempt by the heart to enhance its performance. However, it may become maladaptive when the heart is persistently exposed to an increased load. In cultured H9c2 cells, cell hypertrophy was induced by incubating cells with Ang II. Using rhodamine-conjugated phalloidin staining, cell hypertrophy was investigated. The results revealed that 

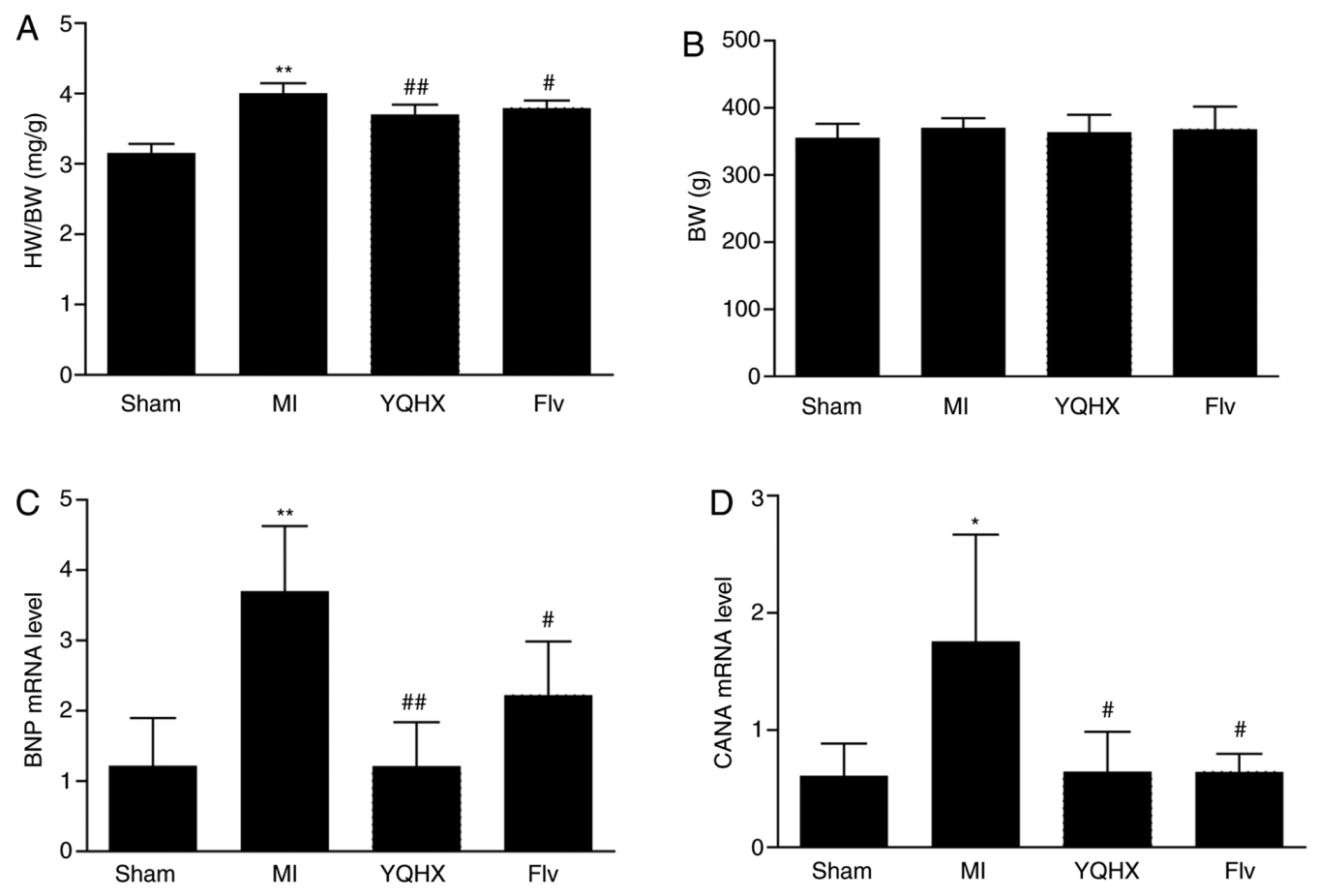

Figure 2. Effect of YQHX and Flv on myocardial hypertrophy in MI rats. (A) HW/BW ratio of rats in each group (n=7-8). (B) BW of rats in each group (n=8). (C) Calcineurin A mRNA levels ( $n=5)$ and (D) BNP mRNA levels $(n=4-5)$ detected by reverse transcription-quantitative polymerase chain reaction. Values are provided as the mean \pm standard deviation. ${ }^{*} \mathrm{P}<0.05,{ }^{* *} \mathrm{P}<0.01$ vs. sham group; ${ }^{*} \mathrm{P}<0.05,{ }^{\# \#} \mathrm{P}<0.01$ vs. MI group. YQHX, Yiqi Huoxue; Flv, fluvoxamine; MI, myocardial infarction; HW, heart weight; BW, body weight; CANA, calcineurin A; BNP, brain natriuretic peptide.

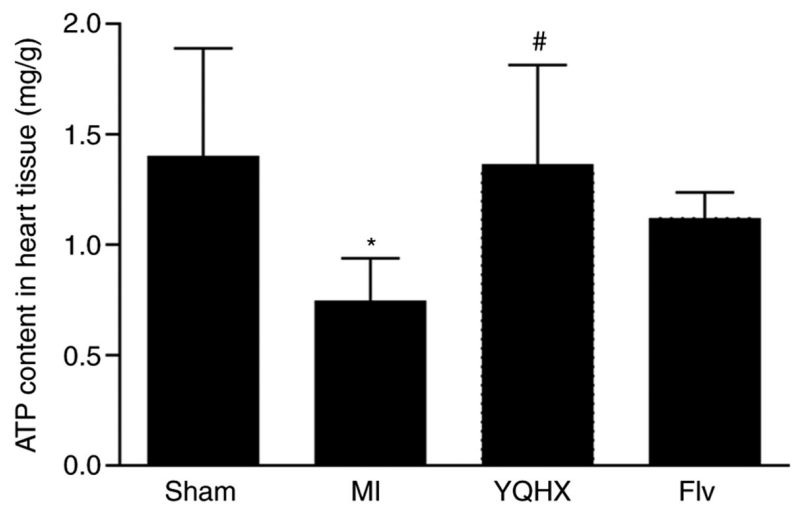

Figure 3. Effect of YQHX and Flv on the ATP content in the heart tissues of MI rats. Values are provided as the mean \pm standard deviation $(n=5)$. ${ }^{*} \mathrm{P}<0.05$ vs. sham group; ${ }^{\text {}} \mathrm{P}<0.05$ vs. MI group. YQHX, Yiqi Huoxue; Flv, fluvoxamine; MI, myocardial infarction.

the cell surface area in Ang II-treated cells was increased significantly compared with control cells $(\mathrm{P}<0.01)$. YQHX treatment attenuated Ang II-induced hypertrophy (Fig. 6A). Using western blotting, Sigma-1R expression was revealed to be downregulated following treatment with Ang II (Fig. 6C), and was the same as reported in a previous study (12). When Ang II-induced H9c2 cells were co-incubated with YQHX, decreased Sigma-1R expression was restored (Fig. 6C). The protein levels of $\mathrm{IP}_{3} \mathrm{R} 2$ were also evaluated by western blotting. The results demonstrated that $\mathrm{IP}_{3} \mathrm{R} 2$ levels were increased in Ang II-incubated cells compared with the control cells and were partially antagonized by YQHX (Fig. 6C). ATP starvation is a pathophysiological characteristic in HF (5).
Our previous in vivo experiments demonstrated that YQHX improved heart energy metabolism in HF rats, indicated by an increased ATP content in heart tissues (Fig. 3). Next, the effect of YQHX on ATP content was investigated in cultured $\mathrm{H} 9 \mathrm{c} 2$ cells and it was demonstrated that cells incubated with Ang II had a decreased ATP content (Fig. 6D), in addition to an increased cell surface area (Fig. 6A).

To determine whether Sigma-1R was the key regulator in the anti-hypertrophy effect of YQHX, H9c2 cells were transfected with a specific inhibitor siRNA of Sigma-1R. As demonstrated in Fig. 6B, the levels of Sigma-1R mRNA expression were downregulated by $65 \%$ in cells incubated with Sigma-1R siRNA $(\mathrm{P}<0.05)$.

These results demonstrated that YQHX treatment attenuated Ang II-induced hypertrophy in Ang II-treated cells. Furthermore, Sigma-1R siRNA was revealed to partially neutralize this effect (Fig. 6A). Additionally, the attenuation of the Ang II-induced decrease in Sigma-1R levels and increase in $\mathrm{IP}_{3} \mathrm{R} 2$ levels by YQHX was inhibited by Sigma-1R siRNA (Fig. 6C), which also attenuated the improvement in the ATP content in Ang II-treated cells by YQHX (Fig. 6D; P>0.05).

\section{Discussion}

In $\mathrm{HF}$, a deficit in energy may account for the contractile dysfunction observed during maximal exertion (5). In traditional Chinese medicine, QiShenYiQi and Qiliqiangxin, which are manufactured using YQHX, have been suggested to ameliorate the symptoms of disorders in cardiac structure and function, possibly via an increase in the myocardial ATP content (20-22). Studies on the major active ingredients of Astragalus, a drug used in the formulation of YQHX, including 


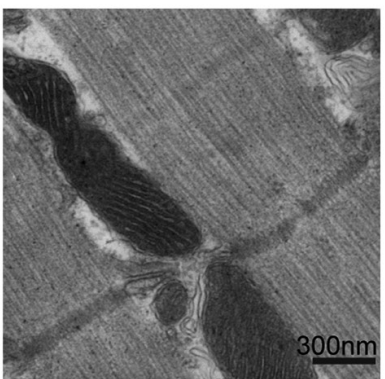

Sham

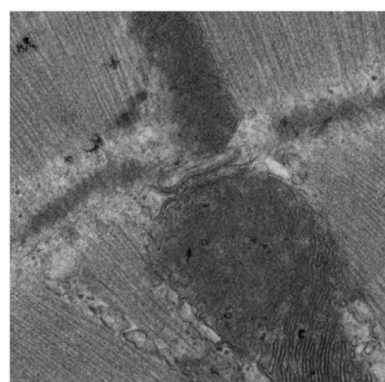

MI

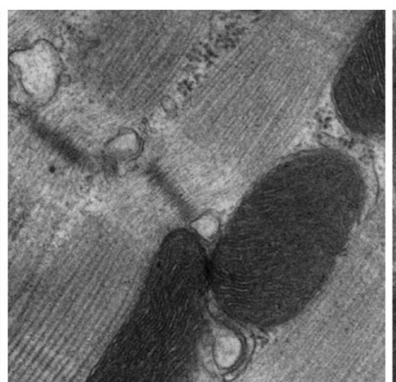

YQHX

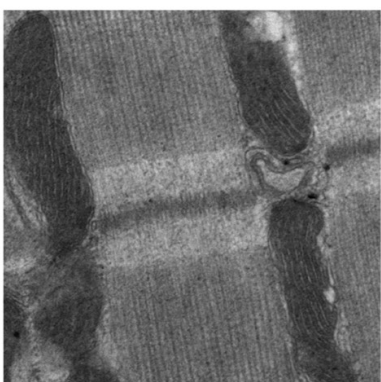

Flv

Figure 4. Representative transmission electron microscopy images of cardiac tissue sections. Scale bar, $300 \mathrm{~nm}$. MI, myocardial infarction; YQHX, Yiqi Huoxue; Flv, fluvoxamine.

A
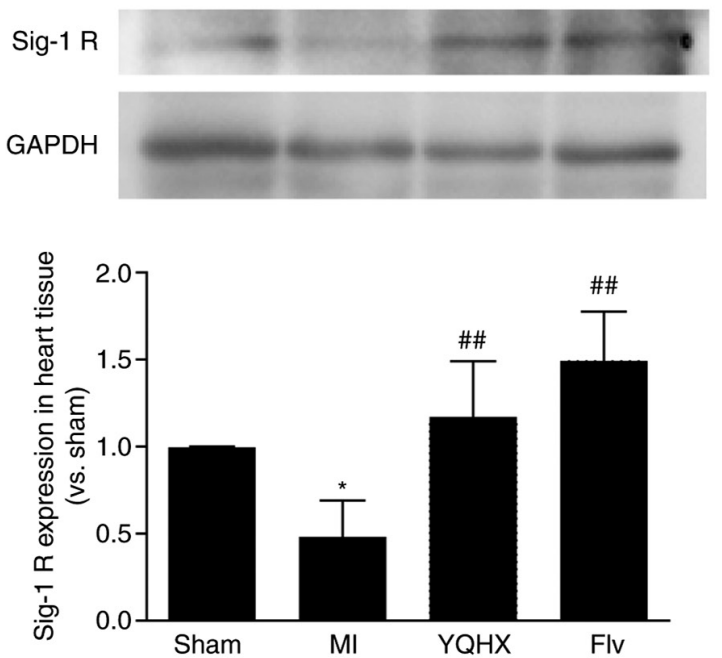

B
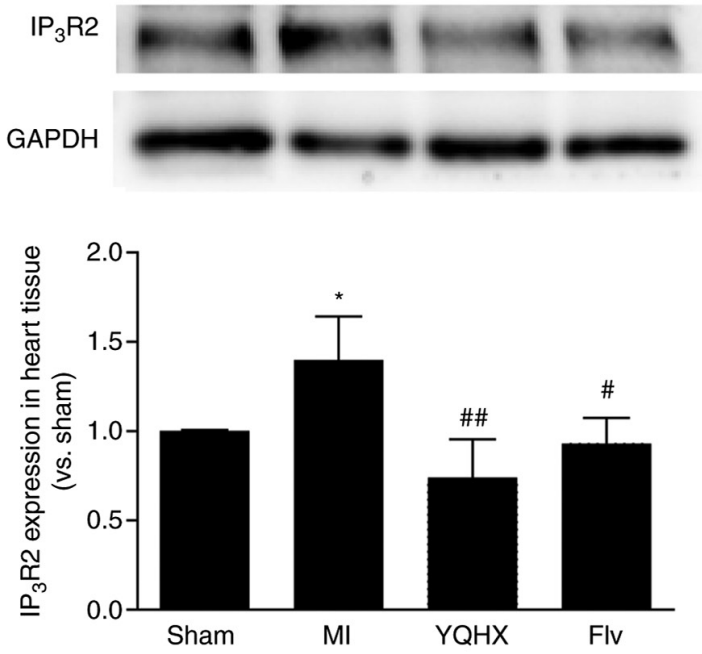

Figure 5. Effect of YQHX and Flv on (A) Sigma-1R and (B) $\mathrm{IP}_{3} \mathrm{R} 2$ expression in the heart tissues in MI rats. Values are provided as mean \pm standard deviation $(\mathrm{n}=4) .{ }^{*} \mathrm{P}<0.05$ vs. sham group; ${ }^{\#} \mathrm{P}<0.05$ vs. MI group; ${ }^{\# \#} \mathrm{P}<0.01$ vs. MI group. MI, myocardial infarction; YQHX, Yiqi Huoxue; Flv, fluvoxamine.

Astragaloside IV and Astragalus polysaccharide, have been proven to regulate mitochondrial function and energy biosynthesis $(23,24)$. In the present study, heart dysfunction in MI rats, represented by decreased LVEF and LVFS, was attenuated after 4 weeks of YQHX treatment. Myocardial hypertrophy, measured as the ratio of HW/BW, was also found to be alleviated following treatment. Taken together, these results indicated that $\mathrm{YQHX}$ improved heart function in MI rats with heart failure, which is consistent with the results of our previous study (18). To investigate the effects of YQHX treatment on energy metabolism in HF, the ATP content and mitochondria ultrastructure were assessed in the heart tissues of MI rats following euthanasia. While the ATP content in rats with HF was decreased, it was found that treatment with YQHX attenuated this effect. Additionally, the impaired mitochondrial ultrastructure of heart tissues post-MI surgery was improved following treatment with YQHX.

Sigma-1R has been demonstrated to participate in numerous diseases, including stroke, dementia and cancer (25), and was recently found to exert cardioprotective effects (12), as well as being identified as a receptor chaperone. It is located in the membrane of MAM, and has been revealed to be expressed abundantly in whole-cell extracts from LV and RV, compared with brain and kidney extracts (7). When Sigma-1R was used as a selective serotonin reuptake inhibitor in a previous study, it was reported to decrease post-MI morbidity and mortality in patients with depression (26). The results of the present study indicated that Sigma-1R expression is decreased in the heart of $\mathrm{HF}$ rats, which is in agreement with the results of a previous study (12). When treated with YQHX, rats with MI surgery showed increased Sigma-1R expression in the heart, as well as increased ATP content and improved heart function. The same change was observed in rats treated with Flv, an agonist of Sigma-1R. Studies have demonstrated that Sigma-1R is associated with the maintenance of mitochondrial structure and function (11). In earlier Sigma-1R $\mathrm{R}^{-/-}$knockdown experiments in mice, irregularly shaped, highly fused mitochondrial network with abnormal cristae and a significantly lower ATP pool were observed in cardiomyocytes (27). In addition, Sigmarl $^{-/-}$hearts showed significant cardiac fibrosis, collagen deposition and the development of cardiac contractile dysfunction (26). The results of the present study suggested that YQHX may improve cardiac function by increasing Sigma-1R expression and improving mitochondrial ultrastructure and ATP production.

Several studies have demonstrated that Sigma-1R serves key roles in heart function improvement by regulating Akt-eNOS signaling and intracellular $\mathrm{Ca}^{2+}(12,28)$. Sigma-1R acts as an inter-organelle signaling modulator between the 
A
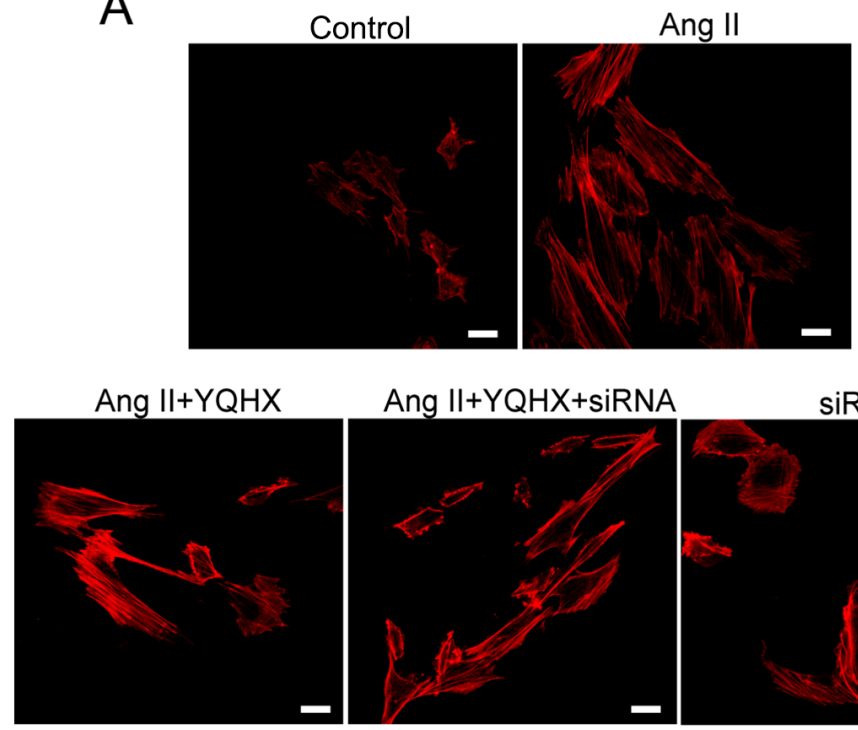

Ang $I I+Y Q H X+s i R N A$
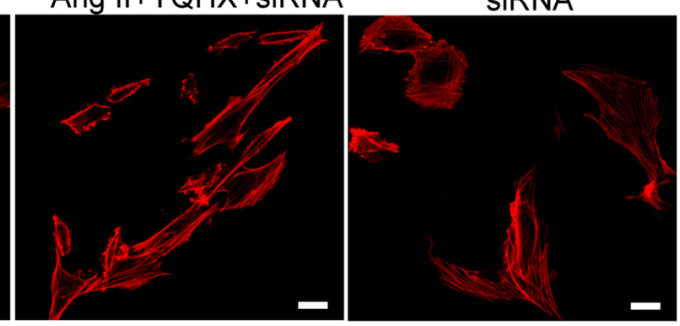

C
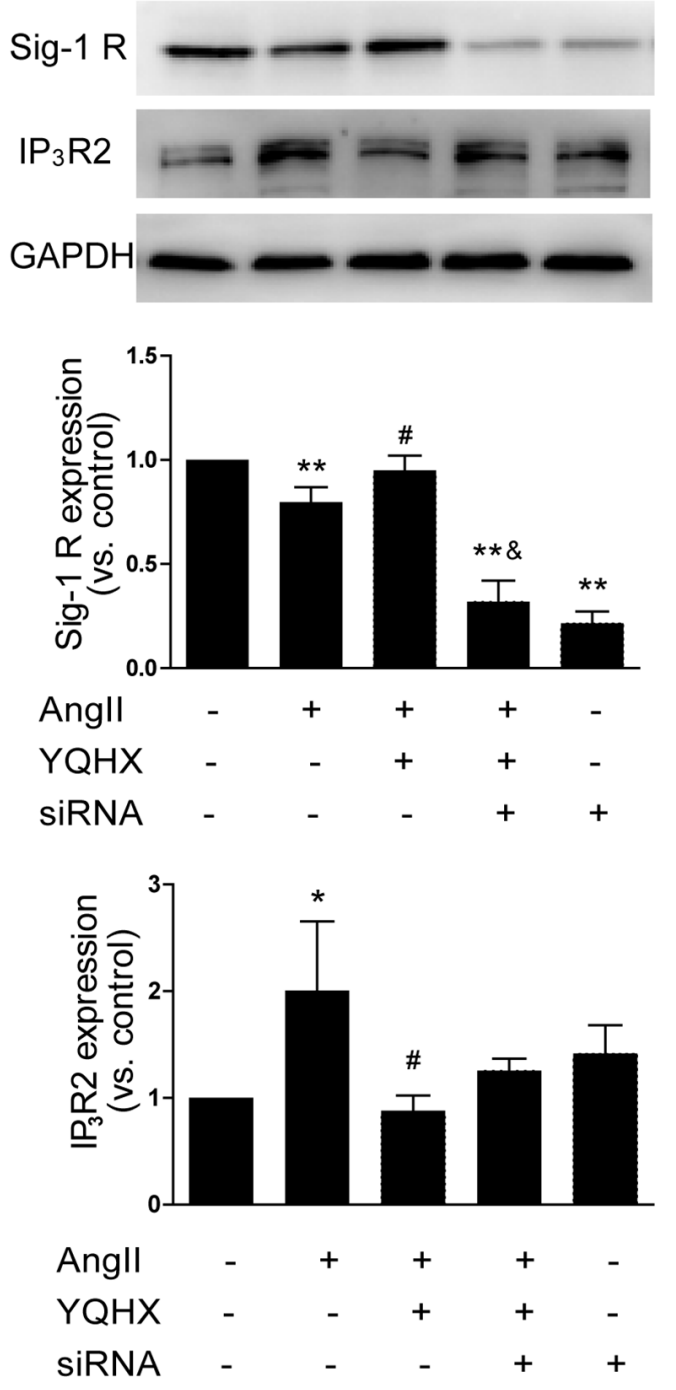

B

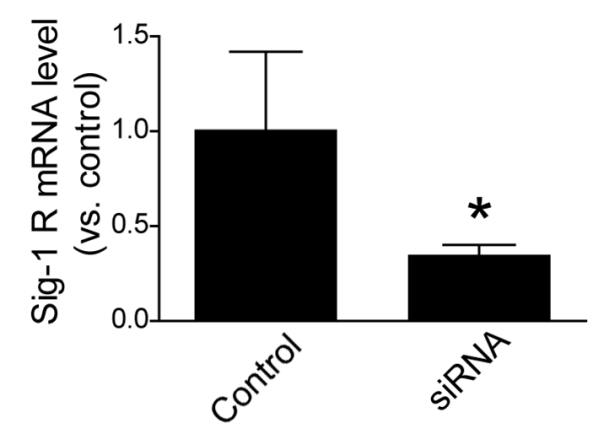

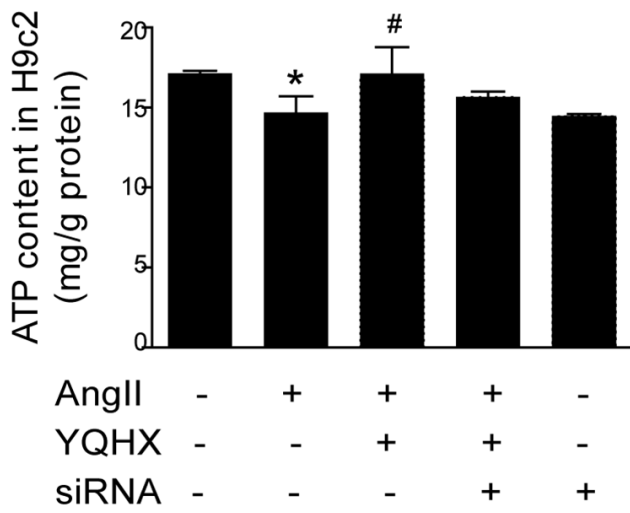

Figure 6. Effect of YQHX on Ang II-induced hypertrophy and ATP decrease in H9c2 cells cultured with Sigma-1R siRNA or negative control siRNA. (A) Laser confocal microscopy photograph of $\mathrm{H} 9 \mathrm{c} 2$ cells stained with rhodamine-conjugated phalloidin for cell hypertrophy and surface area analyzed using Image-Pro Plus 6.0 software. Scale bar, $25 \mu \mathrm{m}$. (B) Sigma-1R mRNA levels detected by reverse transcription-quantitative polymerase chain reaction in H9c2 cells treated with negative control siRNA (control) and Sigma-1R siRNA. (C) Sigma-1R and IP ${ }_{3} 2$ protein expression detected by western blotting in different groups. (D) ATP content in the cultured H9c2 cells in different groups. Values are provided as mean \pm standard deviation $\left(\mathrm{n}=3\right.$ ). ${ }^{*} \mathrm{P}<0.05,{ }^{* *} \mathrm{P}<0.01 \mathrm{vs}$. control group; " $\mathrm{P}<0.05$ vs. Ang II-treated group; ${ }^{\circledR} \mathrm{P}<0.05$ vs. Ang II- and YQHX-treated group. YQHX, Yiqi Huoxue; siRNA, small interfering RNA.

ER and mitochondria, nuclei and plasma membranes. By regulating ER-mitochondrion signaling, Sigma-1R influences intra-mitochondrial $\mathrm{Ca}^{2+}$ homeostasis and cellular bioenergetics (29). Sigma-1R, acting as a molecular chaperone to $\mathrm{IP}_{3} \mathrm{R}$, has been observed to enhance $\mathrm{Ca}^{2+}$ signaling from the ER into mitochondria, thereby activating the tricarboxylic acid 
cycle and increasing ATP production (8). Studies have demonstrated that $\mathrm{IP}_{3} \mathrm{R} 2$ is upregulated and Sigma-1R downregulated in hypertrophic cardiomyocytes. Sigma-1R agonists exhibit improved heart function by stabilizing the Sigma- $1 \mathrm{R}-\mathrm{IP}_{3} \mathrm{R}$ complex (28). In the present study, the levels of $I_{3} R 2$ in the heart tissues of the different groups were detected and it was found that YQHX neutralized the increased $\mathrm{IP}_{3} \mathrm{R} 2$ expression in MI rats. These results suggested that YQHX may improve injured heart function and ATP content in the heart tissue, possibly via Sigma-1R and $\mathrm{IP}_{3} \mathrm{R} 2$ regulation. To confirm the role of Sigma-1R in the cardioprotective effect of YQHX, Sigma-1R was knocked down via siRNA transfection in H9c2 cells in vitro. While YQHX prevented cell hypertrophy and the reduction in ATP induced by Ang II, these positive effects were found to be partially inhibited by Sigma-1R inhibition. These in vitro results suggested that $\mathrm{YQHX}$ is able to increase the ATP content and rescue the cell hypertrophy induced by Ang II, partly by stimulating Sigma-1R. However, further experiments focused on intercellular $\mathrm{Ca}^{2+}$ homeostasis regulation and Sigma-1R-IP ${ }_{3} \mathrm{R}$ complex stabilization are required to confirm these results. Notably, in addition to $\mathrm{IP}_{3} \mathrm{R}$ regulation, Sigma-1R binds to another important $\mathrm{Ca}^{2+}$ transporter-the ryanodine receptor (RyR) and regulates its function (13). Sigma-1R stimulation inhibits the RyR-induced $\mathrm{Ca}^{2+}$ release from the sarcoplasmic reticulum, leading to the suppression of overconstriction (30). In future studies, the mechanisms underlying regulation of $\mathrm{Ca}^{2+}$ homeostasis by Sigma-1R and how YQHX affects this will be investigated.

A previous study demonstrated that the expression of Sigma-1R is regulated by numerous factors, including cold stimulation, ERS signal molecules and certain chemicals (31). A recent study has demonstrated that miRNA may also regulate the expression of Sigma-1R (32). In the present study, numerous monomers in the formulation for YQHX that had effects on the regulation of mitochondrial bioenergetic- $\mathrm{s}$ were investigated (33-35). However, how specific components of YQHX regulate the expression of Sigma-1R requires further study.

In conclusion, these in vivo and in vitro results demonstrated that YQHX improved heart function by regulating the heart's energy metabolism and inhibiting cell hypertrophy. These effects partially relied on the stimulation of Sigma-1R. The results of the present study provided evidence of Sigma- $1 \mathrm{R}$ as a therapeutic target for $\mathrm{HF}$ treatment in traditional Chinese medicine, and the significant therapeutic potential of Sigma-1R for cardiovascular disease. However, further investigation is required to fully elucidate the details of Sigma-1R function in the treatment of HF with YQHX (28).

\section{Acknowledgements}

Not applicable.

\section{Funding}

This study was supported by the National Natural Science Foundation of China (grant nos. 81403366 and 81570656), Fundamental Research Funds for the Central Universities (grant no. 2020-JYB-ZDGG-114) and the 'Xin Ao' research fund of the Beijing University of Chinese Medicine (grant no. 2017-XAJLJJ-012).

\section{Availability of data and materials}

The datasets used and/or analyzed during the current study are available from the corresponding author on reasonable request.

\section{Authors' contributions}

LL, CL, JW, AW, TZ, ZM, YZ, QJ and HC performed the study; LL, LC, DZ and WJL designed the experiments; LL, $\mathrm{BN}$ and WJL analyzed the data; LL and WJL wrote the manuscript. All authors read and approved the final manuscript.

\section{Ethics approval and consent to participate}

All animal procedures were performed in accordance with the guidelines of the Institutional Animal Care and Use Committee of the Beijing University of Chinese Medicine, China.

\section{Patient consent for publication}

Not applicable.

\section{Competing interests}

The authors declare that they have no competing interests.

\section{References}

1. Ambrosy AP, Fonarow GC, Butler J, Chioncel O, Greene SJ, Vaduganathan M, Nodari S, Lam CSP, Sato N, Shah AN and Gheorghiade M: The global health and economic burden of hospitalizations for heart failure: Lessons learned from hospitalized heart failure registries. J Am Coll Cardiol 63: 1123-1133, 2014.

2. Zhang Y, Bauersachs $\mathbf{J}$ and Langer HF: Immune mechanisms in heart failure. Eur J Heart Fail 19: 1379-1389, 2017.

3. Zhou B and Tian R: Mitochondrial dysfunction in pathophysiology of heart failure. J Clin Invest 128: 3716-3726, 2018.

4. Hein S, Arnon E, Kostin S, Schönburg M, Elsässer A, Polyakova V, Bauer EP, Klövekorn WP and Schaper J: Progression from compensated hypertrophy to failure in the pressure-overloaded human heart: Structural deterioration and compensatory mechanisms. Circulation 107: 984-991, 2003.

5. Neubauer S: The failing heart-an engine out of fuel. N Engl J Med 356: 1140-1151, 2007.

6. Ehmke H: The Sigma-1 receptor: A molecular chaperone for the heart and the soul? Cardiovasc Res 93: 6-7, 2012.

7. Bhuiyan MS and Fukunaga K: Targeting Sigma-1 receptor signaling by endogenous ligands for cardioprotection. Expert Opin Ther Targets 15: 145-155, 2011.

8. Hayashi T and Su TP: Sigma-1 receptor chaperones at the ER-mitochondrion interface regulate $\mathrm{Ca}(2+)$ signaling and cell survival. Cell 131: 596-610, 2007.

9. Bhuiyan MS, Tagashira H, Shioda N and Fukunaga K: Targeting Sigma-1 receptor with fluvoxamine ameliorates pressure-overload-induced hypertrophy and dysfunctions. Expert Opin Ther Targets 14: 1009-1022, 2010.

10. Ela C, Barg J, Vogel Z, Hasin Y and Eilam Y: Sigma receptor ligands modulate contractility, $\mathrm{Ca}^{++}$influx and beating rate in cultured cardiac myocytes. J Pharmacol Exp Ther 269: 1300-1309, 1994.

11. Marriott KS, Prasad M, Thapliyal V and Bose HS: $\sigma-1$ receptor at the mitochondrial-associated endoplasmic reticulum membrane is responsible for mitochondrial metabolic regulation. J Pharmacol Exp Ther 343: 578-586, 2012.

12. Tagashira H, Bhuiyan S, Shioda N, Hasegawa H, Kanai H and Fukunaga K: Sigma1-receptor stimulation with fluvoxamine ameliorates transverse aortic constriction-induced myocardial hypertrophy and dysfunction in mice. Am J Physiol Heart Circ Physiol 299: H1535-H1545, 2010. 
13. Tagashira $\mathrm{H}$, Bhuiyan MS and Fukunaga K: Diverse regulation of IP3 and ryanodine receptors by pentazocine through o1-receptor in cardiomyocytes. Am J Physiol Heart Circ Physiol 305: H1201-H1212, 2013.

14. Li XQ, He JC, Huang PX and Cao XB: Chinese medicine syndromes in congestive heart failure: A literature study and retrospective analysis of clinical cases. Chin J Integr Med 22: 738-744, 2016

15. Lin SS, Liu CX, Zhang JH, Wang XL and Mao JY: Efficacy and safety of oral Chinese patent medicine combined with conventional therapy for heart failure: An overview of systematic reviews. Evid Based Complement Alternat Med 2020: 8620186, 2020.

16. Chen YY, Li Q, Pan CS, Yan L, Fan JY, He K, Sun K, Liu YY, Chen QF, Bai Y, et al: QiShenYiQi Pills, a compound in Chinese medicine, protects against pressure overload-induced cardiac hypertrophy through a multi-component and multi-target mode. Sci Rep 5: 11802, 2015.

17. Wang Y, Fu M, Wang J, Zhang J, Han X, Song Y, Fan Y, Hu K, Zhou J and Ge J: Qiliqiangxin improves cardiac function through regulating energy metabolism via HIF-1 $\alpha$-dependent and independent mechanisms in heart failure rats after acute myocardial infarction. Biomed Res Int 2020: 1276195, 2020.

18. Lou LX, Wu AM, Zhang DM, Wu SX, Gao YH, Nie B, Zhao MJ, Lv XY, Jin QS, Zhao YZ, et al: Yiqi Huoxue recipe improves heart function through inhibiting apoptosis related to endoplasmic reticulum stress in myocardial infarction model of rats. Evid Based Complement Alternat Med 2014: 745919, 2014.

19. Livak KJ and Schmittgen TD: Analysis of relative gene expression data using real-time quantitative PCR and the 2 (-Delta Delta C(T)) method. Methods 25: 402-408, 2001.

20. Li J, Guan XK and Liu RX: Role of Chinese herbal medicines in regulation of energy metabolism in treating cardiovascular diseases. Chin J Integr Med 25: 307-315, 2019.

21. Zhang J, Wei C, Wang H, Tang S, Jia Z, Wang L, Xu D and $\mathrm{Wu}$ Y: Protective effect of qiliqiangxin capsule on energy metabolism and myocardial mitochondria in pressure overload heart failure rats. Evid Based Complement Alternat Med 2013: 378298, 2013.

22. Tang DX, Zhao HP, Pan CS, Liu YY, Wei XH, Yang XY, Chen YY, Fan JY, Wang CS, Han JY and Li PP: QiShenYiQi Pills, a compound Chinese medicine, ameliorates doxorubicin-induced myocardial structure damage and cardiac dysfunction in rats. Evid Based Complement Alternat Med 2013: 480597, 2013.

23. Lu Y, Li S, Wu H, Bian Z, Xu J, Gu C, Chen X and Yang D: Beneficial effects of astragaloside IV against angiotensin II-induced mitochondrial dysfunction in rat vascular smooth muscle cells. Int J Mol Med 36: 1223-1232, 2015.

24. Luan A, Tang F, Yang Y, Lu M, Wang H and Zhang Y: Astragalus polysaccharide attenuates isoproterenol-induced cardiac hypertrophy by regulating TNF- $\alpha$-PGC-1 $\alpha$ signaling mediated energy biosynthesis. Environ Toxicol Pharmacol 39: 1081-1090, 2015.
25. Tsai SY,Hayashi T, Mori T and Su TP: Sigma-1 receptor chaperones and diseases. Cent Nerv Syst Agents Med Chem 9: 184-189, 2009.

26. Taylor CB, Youngblood ME, Catellier D, Veith RC, Carney RM, Burg MM, Kaufmann PG, Shuster J, Mellman T, Blumenthal JA, et al: Effects of antidepressant medication on morbidity and mortality in depressed patients after myocardial infarction. Arch Gen Psychiatry 62: 792-798, 2005.

27. Abdullah CS, Alam S, Aishwarya R, Miriyala S, Panchatcharam M, Bhuiyan MAN, Peretik JM, Orr AW, James J, Osinska $\mathrm{H}$, et al: Cardiac dysfunction in the sigma 1 receptor knockout mouse associated with impaired mitochondrial dynamics and bioenergetics. J Am Heart Assoc 7: e009775, 2018.

28. Tagashira H, Bhuiyan MS, Shioda N and Fukunaga K: Fluvoxamine rescues mitochondrial $\mathrm{Ca}^{2+}$ transport and ATP production through $\sigma(1)$-receptor in hypertrophic cardiomyocytes. Life Sci 95: 89-100, 2014.

29. Su TP, Hayashi T, Maurice T, Buch S and Ruoho AE: The Sigma-1 receptor chaperone as an inter-organelle signaling modulator. Trends Pharmacol Sci 31: 557-566, 2010.

30. Kushnir A, Wajsberg B and Marks AR: Ryanodine receptor dysfunction in human disorders. Biochim Biophys Acta Mol Cell Res 1865: 1687-1697, 2018.

31. Rousseaux CG and Greene SF: Sigma receptors [sigmaRs]: Biology in normal and diseased states. J Recept Signal Transduct Res 36: 327-388, 2016.

32. Bao Q, Zhao M, Chen L, Wang Y, Wu S, Wu W and Liu X: MicroR NA-297 promotes cardiomyocyte hypertrophy via targeting Sigma-1 receptor. Life Sci 175: 1-10, 2017.

33. Liu Y, Hu Y, Qiukai E, Zuo J, Yang L and Liu W: Salvianolic acid B inhibits mitochondrial dysfunction by up-regulating mortalin. Sci Rep 7: 43097, 2017.

34. Kwan KK, Huang Y, Leung KW, Dong TT and Tsim KW: Danggui Buxue Tang, a Chinese herbal decoction containing astragali radix and angelicae sinensis radix, modulates mitochondrial bioenergetics in cultured cardiomyoblasts. Front Pharmacol 10: 614, 2019.

35. Jin HJ and Li CG: Tanshinone IIA and cryptotanshinone Prevent mitochondrial dysfunction in hypoxia-induced $\mathrm{H} 9 \mathrm{c} 2$ cells: Association to mitochondrial ROS, intracellular nitric oxide, and calcium levels. Evid Based Complement Alternat Med 2013: 610694, 2013.

This work is licensed under a Creative Commons Attribution-NonCommercial-NoDerivatives 4.0 International (CC BY-NC-ND 4.0) License. 